\title{
Does Energy Expenditure Affect the Perception of Egocentric Distance? A Failure to Replicate Experiment 1 of Proffitt, Stefanucci, Banton, and Epstein (2003)
}

\author{
Jeffrey J. Hutchison and Jack M. Loomis \\ University of California
}

\begin{abstract}
In a series of recent studies, Proffitt and his colleagues have reported that the perceived distance to a target is influenced by the energy expenditure associated with any action, such as walking or throwing, for spanning the distance to the target. In particular, Proffitt, Stefanucci, Banton, and Epstein (2003) reported that wearing a heavy backpack caused verbal reports of distance to increase. We conducted a study to determine whether three responses dependent on perceived distance (verbal report of distance, blind walking, and estimates of object size) are influenced by the backpack manipulation. In two experiments, one involving a between-participants design and the other involving a within-participants design, we found that none of the three responses were influenced by the wearing of a heavy backpack.
\end{abstract}

Keywords: distance perception, energy expenditure

En una serie reciente de trabajos, Proffitt y sus colegas informaron de que la distancia a la que se percibe una estimulación diana se ve afectada por el gasto de energía asociado a la realización de cualquier acción, como andar o lanzar un objeto, que pueda realizarse para cubrir la distancia hasta la estimulación diana. Concretamente, Proffitt, Stefanucci, Banton y Epstein (2003) afirmaron que llevar una mochila pesada hizo que se incrementasen los informes verbales sobre la distancia. Realizamos un estudio para verificar si tres respuestas que dependen de la distancia percibida (informe verbal de distancia, andar a ciegas y estimaciones del tamaño de un objeto) son afectadas por el uso de la mochila. En dos experimentos, uno con un diseño inter-participantes y el otro con un diseño intra-participantes, encontramos que ninguna de las tres respuestas era afectada por llevar una mochila pesada.

Palabras clave: percepción de la distancia, gasto de energía

This research was supported by a grant from the Department of Undergraduate Research and Creative Activities at the University of California, Santa Barbara.

A debt of gratitude is owed to the following people for their contributions to this research: Art Gilbert and Jim Romeo for their guidance and elucidation of energetics, Joe Jablonski for assistance with apparatus, and Kristen Macuga for assistance with the statistical analysis.

Correspondence concerning this arcitle should be addressed to Jeffrey J. Hutchison, Department of Cognitive \& Linguistic Sciences, Brown University, Providence, RI 02192 (USA). 
In a series of recent papers, Proffitt and his colleagues have provided evidence that the visually perceived distance to a target is influenced both by the energy expenditure required to carry out an action spanning that distance and by the physiological state of the person (Bhalla \& Proffitt, 1999; Proffitt, Bhalla, Gossweiler, \& Midgett, 1995; Profitt, Stefanucci, Banton, \& Epstein, 2003; Witt, Proffitt, \& Epstein, 2004). In particular, Profitt et al. (2003) reported that observers, who wore a heavy backpack while judging the distance to a target with the expectation of having to walk to it while wearing the backpack, reported greater distances than participants who did not wear backpacks. These various results indicate that the energy expenditure $^{1}$ of performing an action influences the perceived distance to be spanned by the action. In addition, these and other researchers (e.g., Creem-Regehr, Gooch, Sahm, \& Thompson, 2004) argue that the representation of physical space is action specific. If throwing rather than walking is used to span the distance to the target, only energy expenditure manipulations that influence the intended action will affect the perceived distance.

The effect of energy expenditure on perceived egocentric distance, if true, is very important. The conventional view among space perception researchers has been that perceived visual space is a consciously experienced internal representation of physical space and that egocentric distance within it can be measured using a variety of different responses (e.g., Loomis \& Knapp, 2003). According to this view, there are a number of converging measures of egocentric distance perception, some based on action and others not, that should all show the same effects of any manipulation predicted to alter perceived distance. Action based measures of perceived egocentric distance include ball throwing, blind walking, triangulation methods, and more recently a measure based on a response in 3-D space consisting of blind walking and a pointing gesture by the hand (Ooi, Wu, \& He, 2001). Non-action based measures include verbal report of perceived distance, an estimate of perceived distance derived from distal size judgments by way of size-distance invariance (e.g., Gogel, Loomis, Newman, \& Sharkey, 1985), and estimates of distance derived from judgments of shape by way of the coupling observed between perceived shape and perceived distance (Ooi, Wu, \& He, in press; Wu, Ooi, \& He, 2004).

The two experiments reported here were intended first to replicate the effect of the backpack manipulation on verbal reports of distance, as reported by Proffitt et al. (2003), and then to determine whether two additional responses dependent upon perceived distance are similarly affected. Unfortunately, we were unable to replicate the basic effect of the backpack manipulation on any of the three responses, which leaves moot the question of whether the backpack manipulation indeed modifies perceived egocentric distance, as assessed using converging measures.

Experiment 1 used a between-participants design to assess the effect of the backpack manipulation, following closely the design of Proffitt et al. (2003). There were two experimental manipulations. The first was the backpack manipulation of Proffitt et al. Putting on a heavy backpack was reported to increase perceived distance, as indicated by increases in verbally reported distance. The second manipulation was a restriction of the participant's field of view, which causes a reduction of perceived distance. Upon finding no effect of the backpack manipulation in Experiment 1 using the between-participants design, we attempted to increase the power of our manipulations by switching to a within-participants design in Experiment 2, but the same null result was obtained.

With the two manipulations of Experiment 1, there were three experimental conditions. The control condition involved viewing spheres of different sizes at different distances, under full cue conditions. Participants verbally estimated the egocentric distance and diameter of each target sphere. Participants then performed an indirect blind walking task (also called "triangulated walking") to the various targets (Loomis, Klatzky, Philbeck, \& Golledge, 1998; Philbeck, Loomis, \& Beall, 1997; Thompson et al., 2004). Participants viewed the target and then, while wearing a blindfold, first walked along a straight path oblique to the target and then turned and walked the rest of the way to the estimated target location. In the backpack condition, participants viewed targets under full cue viewing conditions while wearing by a backpack and then made the three responses while wearing the backpack. This condition is essentially a replication of the experiment by Proffitt et al. (2003), except for the addition of the size judgments and blind walking task. Based on the work of Proffitt et al., the backpack manipulation was expected to produce increases in perceived distance. Other than the energy expenditure and physiological state manipulations of Proffitt and his colleagues, we were unaware of manipulations in real environments that produce increases of distance estimates under full cue conditions. Therefore, we chose to use instead a manipulation for reducing perceived distance. To effect this reduction, we greatly restricted the field of view on the participants, a manipulation found to decrease perceived egocentric distance, as measured using variants of blind walking (Creem-Regehr, Willemsen,

\footnotetext{
1 For an extended discussion of the meanings of effort, energy expenditure, physiological state, and physiological potential as they relate to space perception, see the first author's Honors Undergraduate Thesis (Hutchison, 2005).
} 
Gooch, \& Thompson, 2005; Wu et al., 2004) $)^{2}$ Thus, participants in the present reduced field of view (RFOV) condition viewed targets through a restrictive aperture and made the same estimates as in the other two conditions. We expected that participants would show reduced estimates of distance using both verbal reports and the blind walking task, relative to the responses in the control condition.

In addition to verbal report and indirect blind walking, we obtained size estimates of the targets, which varied in size. According to the size distance invariance hypothesis $(\mathrm{SDIH})$, perceived size ( $\left.\mathrm{S}^{\prime}\right)$ is a function of both the perceived distance (D') and the angular size of a target $(\alpha)$ : $\mathrm{S}^{\prime}=2 \mathrm{D}^{\prime} \tan (\alpha / 2)$ (Gilinsky, 1951; Sedgwick, 1986). Consequently, for a constant angular size, increases in perceived distance should result in proportional increases in the perceived size. Because of the reported reduction of perceived distance produced by restricting the field of view (Creem-Regehr et al., 2005; Wu et al., 2004), we expected proportional decreases in estimated target size. Similarly, if wearing a backpack increases perceived distance, there should be proportional increases in estimated target size.

\section{Experiment 1}

\section{Method}

\section{Participants}

The participants were 36 undergraduates at the University of California, Santa Barbara. Participants were paid or received credit for an introductory psychology course. All

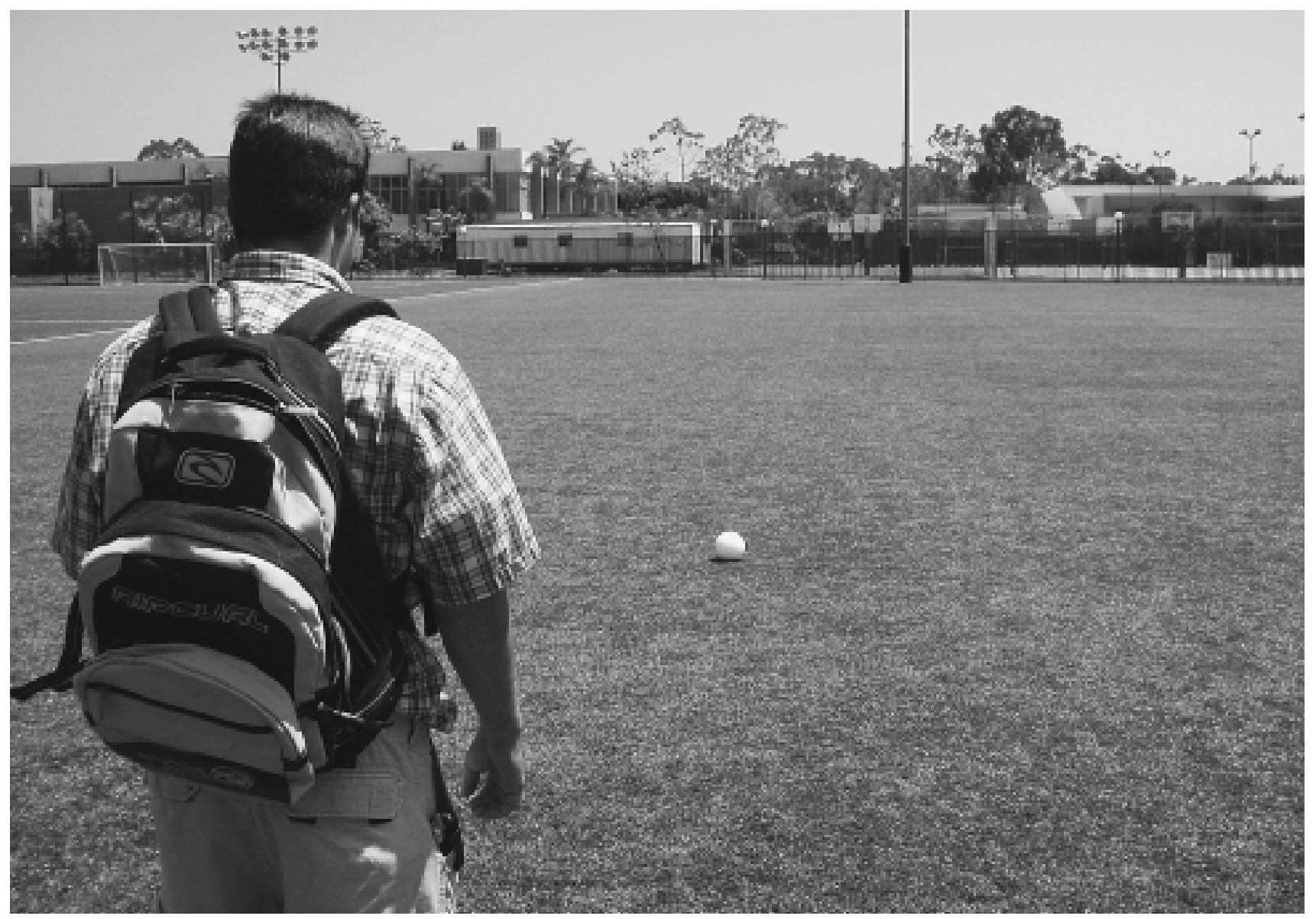

Figure 1. A participant wearing the backpack while viewing one of the target stimuli in the field of artificial turf.

\footnotetext{
2 Restricting the field of view has an appreciable effect on distance perception, especially when the observer is not allowed to look around while wearing the field-restricting aperture (Creem-Regehr et al., 2005; Wu et al., 2004). In contrast, Knapp and Loomis (2004) found no effect of a moderately sized restrictive aperture on distance perception when observers were free to scan the environment with their heads while wearing the aperture. These results are consistent with the hypothesis of a Sequential Surface Integration Process, by which a perceptual representation of a surface is sequentially constructed from successive samples of visual information from the environment (e.g., Ooi et al, in press; Wu et al., 2004)
} 
had normal or corrected to normal vision. They were naïve about the purpose of the experiment. Twelve participants were assigned to each the 3 conditions ( 6 males in the control condition, 6 males in the backpack condition, and 4 males in the RFOV condition).

\section{Apparatus and Stimuli}

Distance and size judgments were made outdoors on a homogenous artificial turf during the middle of the day. The near homogeneity of the ground texture provided no explicit distance cues. Small strips of tape were used to demarcate the various distances used for presenting the targets. The tape was not visible from participants' vantage point. The following distances were used: 3, 5, 7, 9, 10, 11, 13, and $15 \mathrm{~m}$. The distances were presented in the following order: $5,13,3,15,10,7,11$, and $9 \mathrm{~m}$ for each condition.

Four white styrofoam spheres were used as targets. The spheres were $4,5,6$, and 8 inches $(10.2,12.7,15.2$, and $20.3 \mathrm{~cm}$, respectively) in diameter. The viewing order of the spheres in all three conditions was as follows: $6,4,8$, $5,6,4,8$, and 5 .

For the backpack condition, participants wore an ergonomic backpack, with hip strap, loaded with an even distribution of textbooks and standard weight plates, so that the total weight was between 1/5 and 1/6 of their reported body weight (Figure 1). For the RFOV condition, an apparatus, originally used in a study by Knapp and Loomis (2004), was modified to further restrict the participant's field of view (Figure 1). The apparatus was constructed of poster board, felt and safety goggles. The binocular field of view was $51.6^{\circ}$ degrees horizontal by $18.3^{\circ}$ vertical. The horizontal field of view of each eye extended slightly beyond the stated value.

\section{Procedure}

Prior to the experiment proper, participants filled out a brief questionnaire answering questions about university major, year in school, gender, height, and, most importantly, weight. The reported weight was used to increase the weight of a backpack for those participants in the backpack condition.

Once the experiment was underway, participants were blindfolded and presented with music over earphones in order to eliminate any auditory cues during target placement. During target presentation, the blindfold and earphones were displaced and participants viewed the targets and, at their leisure, gave verbal estimates of its distance (in feet) and diameter (in inches). Participants in the RFOV condition remained blindfolded until they were placed at the restricted field of view aperture. Participants were instructed to place their forehead against the rim of the safety goggles prior to removing the blindfold. They were never allowed to view the target and ground texture other than through the aperture. After the distance and size estimates in all conditions, the blindfold was then replaced and participants walked along an indirect path to the target location; the earphones were replaced while the target was moved out of the way, but participants took them off prior to walking.. For those participants in the RFOV condition, the viewing apparatus was first moved aside. Participants in the backpack condition continued wearing the backpack while walking. Participants walked along a straight path oblique to the target (by about $30^{\circ}$ ) and upon a verbal command from the experimenter, turned toward the target and continued walking until they had reached the estimated target location. The target was removed prior to walking so that participants had no possibility of collision. The stopping locations on each trial were marked on the ground. After all trials were completed, a tape measure was used to measure the distances from the stopping point to each of two locations on either side of the viewing location. These two distances for each stopping location were used to compute, by way of trilateration, the coordinates of the stopping location relative to the viewing location (see Fukusima, Loomis, \& Da Silva, 1997). In turn, the direct distance from the viewing location to the stopping location could be computed.

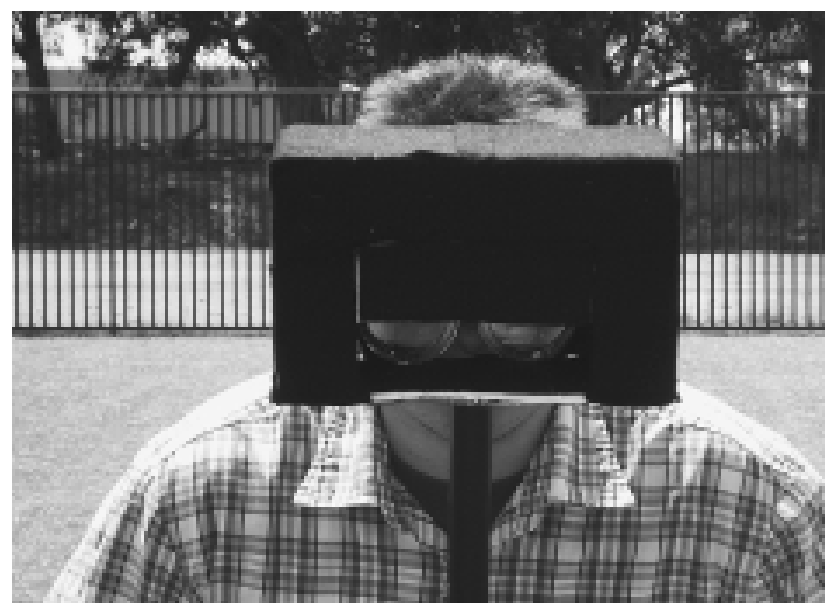

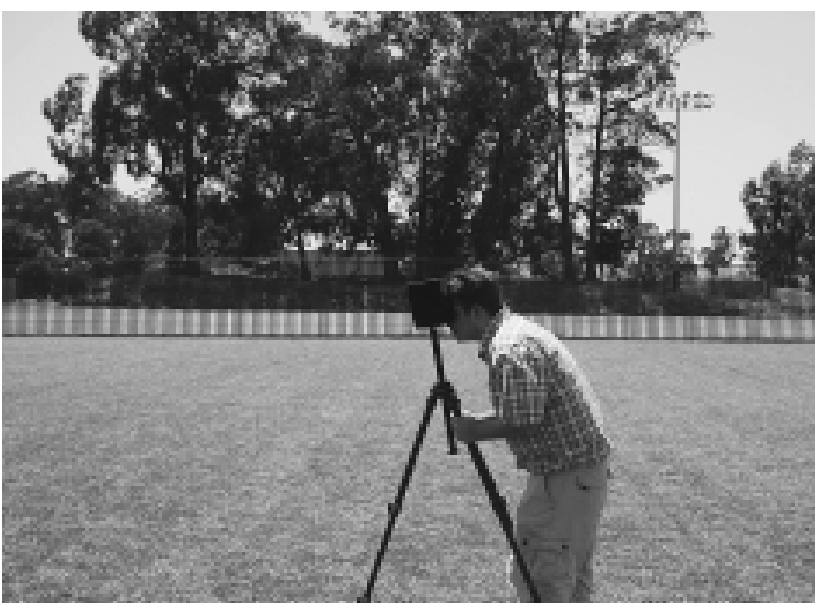

Figure 2. Two views of a participant viewing through the restrictive aperture. 


\section{Results}

\section{Verbal Reports of Distance}

The mean distance estimates in each condition are given in the left panel of Figure 3. Linear regression functions with 0 intercept have been fit to the different data sets. The mean estimates in the control condition were all less than the target distances (slope of the regression function = 0.69), a result consistent with previous research (e.g., Foley, Ribeiro, \& Da Silva, 2004; Kelly, Loomis, \& Beall, 2004; Loomis et al., 1998). A 2 (RFOV vs. Control) $\times 8$ (Distance) mixed-factor ANOVA on the distance estimates revealed that participants in the RFOV condition judged targets to be significantly closer than those in the control condition, $F(1,22)=4.608, p<.05$; the slope of the linear regression function was 0.57 , which is 0.83 times that of the control condition. A 2 (Backpack vs. Control) $\times 8$ (Distance) mixedfactor ANOVA on the distance estimates revealed no significant difference between the backpack and control conditions, $F(1,22)=0.72, p=0.41$, although the trend was in the expected direction (based on the earlier work of Proffitt et al., 2003); the slope of the regression function was 0.79 .

\section{Blind Walking Estimates of Distance}

The mean distances from the viewing point to the stopping point of the indirect blind walking responses are given in the center panel of Figure 3 for all three conditions. A 2 (RFOV vs. Control) $\times 8$ (Distance) mixed-factor ANOVA on the distance responses revealed that participants in the RFOV condition walked to locations significantly closer than those in the control condition, $F(1,22)=4.859$, $p<.05$; the slopes of the best fitting linear regression functions were 0.74 for the RFOV condition and 0.86 for the control condition, with a ratio of 0.86. A 2 (Backpack vs. Control) $\times 8$ (Distance) mixed-factor ANOVA on the distance responses indicated no significant difference between the backpack and control conditions, $F(1,22)=0.567, p$ $=.46$, although the trend was in the expected direction, with a slope of 0.90 for the backpack condition vs. 0.86 in the control condition.

\section{Size Estimates}

Each participant judged the diameter of each of the 4 target spheres twice. Each of the spheres was presented at each of two distances, and this association between sphere size and distance was the same for all participants. The mean estimates of size for the targets, averaged over participants, were calculated without regard to the distances at which the targets were presented under the assumption of size constancy, a special case of size-distance invariance
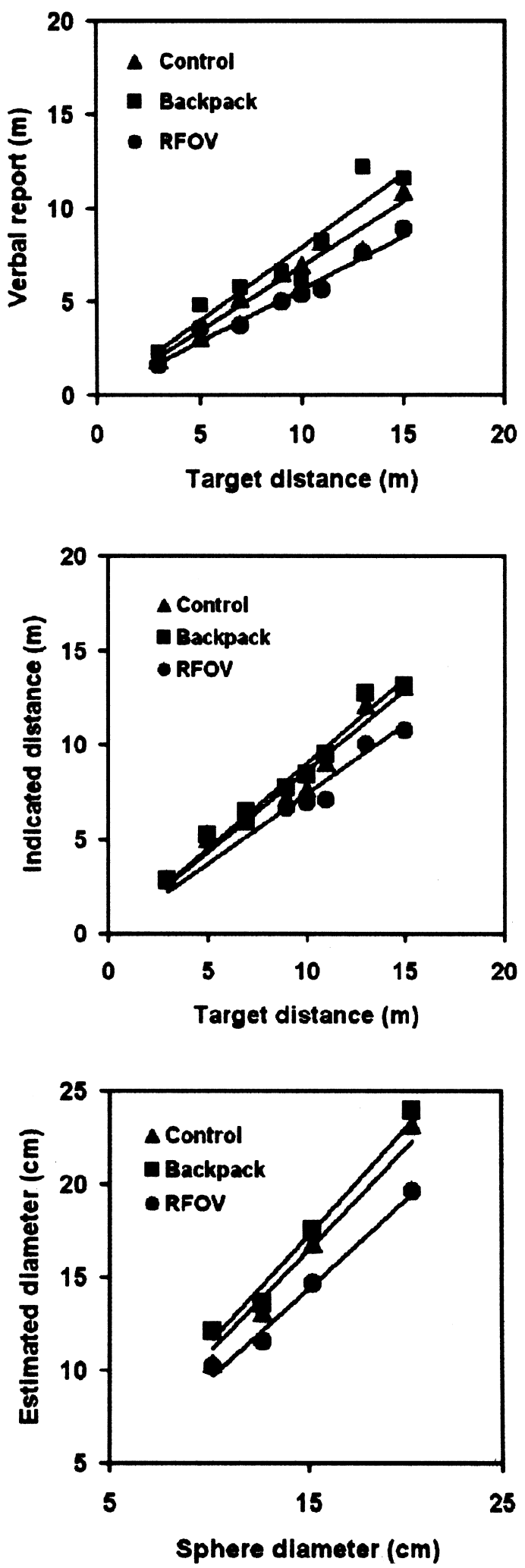

Figure 3. The results of Experiment 1. The left panel gives mean verbal reports of distance as a function of target distance. The center panel gives the mean distances of the stopping points for blind walking from the viewing point, as a function of target distance. The right panel gives the mean estimates of the diameters of the target spheres. 
for which perceived distance is proportional to target distance. The mean estimates of size for the 4 spheres are given in the right panel of Figure 3 for all three conditions. The slope of the best fitting regression function for the RFOV condition was 0.87 times that of the slope for the control condition. A 2 (RFOV vs. Control) $\times 8$ (Target) mixed-factor ANOVA on the size estimates revealed that participants in the RFOV condition judged the targets to be significantly smaller than they did in the control condition, $F(1,22)=5.403, p<.05$. A 2 (Backpack vs. Control) $\times$ 8 (Target) mixed-factor ANOVA on the size estimates revealed no significant difference between the backpack and control conditions, $F(1,22)=0.82, p=.777$.

\section{Discussion}

Based on the previous work of Creem-Regehr et al. (2005) and Wu et al. (2004), it was expected that reducing the participant's field of view would cause a reduction of the perceived distances of the different targets. This result was obtained with both direct measures of perceived distance, verbal report and the distance to the stopping points in the indirect blind walking task; the ratios of the slopes of the regression functions (RFOV/control) were 0.83 and 0.86 for verbal report and blind walking, respectively. In addition, as expected from size-distance invariance, the estimated sizes of the different target spheres were reduced as field of view was reduced (from control to RFOV conditions). The slope of the best fitting regression function for the RFOV condition was 0.87 that of the control condition. This ratio is close to the two corresponding ratios for verbal report and for blind walking, indicating that perceived size was reduced more or less in proportion with the reduction of perceived distance, as expected on the basis of size-distance invariance. The agreement of the three measures is strong evidence that perceived distance is indeed reduced when field of view is reduced.

In sharp contrast, the backpack manipulation had no reliable effect in terms of any of the three response measures. The null effect of the backpack manipulation is a failure to replicate the increase of perceived distance, as reflected in verbal reports, as reported by Proffitt et al. (2003).

Proffitt et al. (2003) used a between-participants design in their experiment. Because we thought that a more robust effect of the backpack manipulation might show up using a within-participants design, we repeated the backpack manipulation in Experiment 2 using such a design. Also, in order to focus on the effect of the backpack manipulation, we did not repeat the RFOV manipulation. Other modifications made in order to improve the efficiency of the experiment were omission of the blind walking responses and the use of a smaller number of target distances.

\section{Experiment 2}

\section{Method}

\section{Participants}

The participants were 12 undergraduates (4 male and 8 female) from the University of California, Santa Barbara. Participants were either paid or received credit for an introductory psychology course. All had normal or corrected to normal visual acuity. They were naïve about the purpose of the experiment.

\section{Apparatus and Stimuli}

The backpack from Experiment 1, weighted with textbooks and standard weight plates, was used once again. This time, participants viewed targets at the following distances in the two conditions of the experiment (control and backpack): 3, 8, 11, and 15 meters. Two dummy distances were also utilized in each condition so as reduce the likelihood that the participants would recognize that the same 4 distances were being used in the two conditions. The dummy distances were randomly selected from the following: 4, 9, 12, and 14 meters. The order of distances was randomly determined for each participant, in contrast with the constant order used in Experiment 1.

The same four spheres were used as targets. Each sphere was assigned to one of the distances used on the non-dummy trials. The viewing order of the spheres was randomly determined for each participant, meaning that different participants did not view the same spheres at the various target distances as they did in Experiment 1.

\section{Procedure}

The procedure for this experiment was similar to that of Experiment 1. As noted, participants did not complete the blind walking task utilized in the previous experiment. Participants made estimates of egocentric distance and size of the targets both with and without the backpack. They were assigned to wear the backpack either during the first block of trials or during the second block.

\section{Results and Discussion}

\section{Verbal Reports}

The mean values of verbal report, averaged over participants, are given in the left pane of Figure 4 for the two conditions. A 2 (Control vs. Backpack) $\times 4$ (Distance) repeated measures analysis of variance, revealed no significant effect of wearing a backpack, $F(1,11)=1.521$, $p=.243$. 

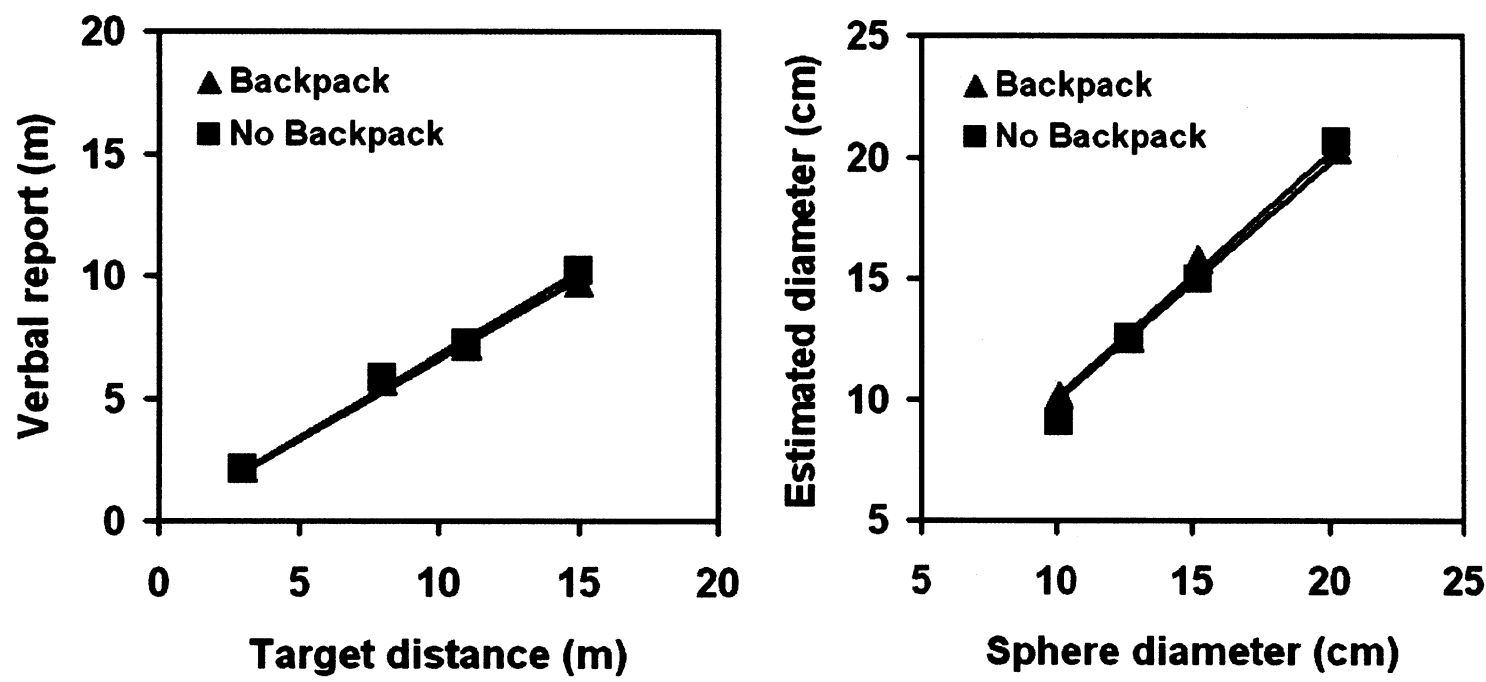

Figure 4. The results of Experiment 2. The left panel gives mean verbal reports of distance as a function of target distance. The right panel gives the mean estimates of the diameters of the target spheres.

\section{Size Estimates}

The mean values of verbal report, averaged over participants, are given in the right panel of Figure 4 for the two conditions. A 2 (Control vs. Backpack) $\times 4$ (Distance) repeated measures analysis of variance, revealed no significant effect of wearing a backpack, $F(1,11)=0.637$, $p=.442$.

Like the between-participants design of Experiment 1, the within-participants design of this experiment failed to produce significant effects of the backpack manipulation on either verbal reports of distance or verbal estimates of size.

\section{General Discussion}

The RFOV manipulation in Experiment 1 produced reductions in perceived distance, as indicated by verbal reports and blind walking responses, and concomitant reductions in perceived size, as expected on the basis of size-distance invariance. The slopes of the regression functions for verbal reports, walking responses, and size estimates in the RFOV condition were $0.83,0.86$, and 0.87 times the corresponding slopes in the control condition. The close agreement of these values is strong evidence that reducing field of view does indeed cause a reduction in perceived egocentric distance.

In contrast, the backpack manipulation in both experiments produced no reliable effect on verbal reports, contrary to the results of Proffitt et al. (2003), nor reliable effects on the indirect blind walking responses and size estimates. Because we were of the opinion that the effect of the backpack manipulation of Proffitt et al. was more likely a judgmental/cognitive effect rather than an effect on perceived visual space, we had anticipated that we would replicate their backpack effect for verbal reports but would find no effect on either the indirect blind walking responses or the size estimates. Because none of our responses showed any reliable effect of the backpack manipulation, we have no evidence on the issue of whether the effect of wearing a backpack, if it exists, is perceptual or postperceptual in nature.

We designed Experiment 1 to be very similar to the experiment of Proffitt et al. (2003), even seeking advice from one of the coauthors in the design stage (J. Stefanucci, personal communication, April 28, 2005). Our failure to replicate the effect of the backpack manipulation, of course, does not negate their result but merely raises questions about its robustness. More research will be needed to determine the conditions under which their effect occurs. More generally, research is needed to examine the claims of Proffitt and his colleagues about the effects of energy expenditure and the physiological state of the participants in order to determine whether these are truly influences on perceived visual space, as they claim, or only influences on postperceptual processes, as we are inclined to believe.

\section{References}

Bhalla, M., \& Proffitt, D.R. (1999). Visual-motor recalibration in perceiving geographical slants. Journal of Experimental Psychology: Human Perception and Performance, 4, 10761096.

Creem-Regehr, S.H., Gooch, A.A., Sahm, C.S., \& Thompson, W.B. (2004). Perceiving virtual geographic slant: Action influences perception. Journal of Experimental Psychology: Human Perception and Performance, 30, 811-821. 
Creem-Regehr, S.H., Willemsen, P., Gooch, A.A., \& Thompson, W.B. (2005). The influence of restricted viewing conditions on egocentric distance perception: Implications for real and virtual indoor environments. Perception, 34, 191-204.

Foley, J.M., Ribeiro, N.P., \& Da Silva J.A. (2004). Visual perception of extent and the geometry of visual space. Vision Research, 44, 147-156.

Fukusima, S.S., Da Silva, J.A., \& Loomis, J.M. (1997). Visual perception of egocentric distance as assessed by triangulation. Journal of Experimental Psychology: Human Perception and Performance, 23, 86-100

Gilinsky, A.S. (1951). Perceived size and distance in visual space. Psychological Review, 58,460-482.

Gogel, W.C., Loomis, J.M., Newman, N.J., \& Sharkey, T.J. (1985). Agreement between indirect measures of perceived distance. Perception \& Psychophysics, 37, 17-27.

Hutchison, J.J. (2005). The effect of physiological potential on egocentric distance and size perception. Honors undergraduate thesis, Department of Psychology, University of Califorinia.

Kelly, J.W., Loomis, J.M., \& Beall, A.C. (2004). Judgments of exocentric distance in large-scale space. Perception, 33, 443-454.

Knapp, J.M., \& Loomis, J.M. (2004). Limited field of view of headmounted displays is not the cause of distance underestimation in virtual environments. Presence, 13, 572-577.

Loomis, J.M., Klatzky, R.L., Philbeck, J.W., \& Golledge, R.G. (1998). Assessing auditory distance perception using perceptually directed action. Perception and Psychophysics, 60, 966-980.

Loomis, J.M. \& Knapp, J.M. (2003). Visual perception of egocentric distance in real and virtual environments. In L.J. Hettinger \& M.W. Haas (Eds.), Virtual and adaptive environments (pp. 21-46). Mahwah, NJ: Erlbaum.
Ooi, T.L., Wu, B., \& He, Z J. (2001). Distance determined by the angular declination below the horizon. Nature, 414, 197-200.

Ooi, T.L., Wu, B., \& He, Z.J. (in press). Perceptual space in the dark affected by the intrinsic bias of the visual system. Perception.

Philbeck, J.W., Loomis, J.M., \& Beall, A.C. (1997). Visually perceived location is an invariant in the control of action. Perception and Psychophycsics, 59, 601-602.

Proffitt, D.R., Bhalla, M., Gossweiler, R., \& Midgett J. (1995). Perceiving geographical slant. Psychonomic Bulletin and Review, 2, 409-428.

Proffitt, D.R., Stefanucci, J., Banton, T., \& Epstein, W. (2003). The role of effort in distance perception. Psychological Science, 14, 106-113.

Sedgwick, H.A. (1986). Space perception. In K.R. Boff, L. Kaufman, \& J.P. Thomas (Eds.), Handbook of perception and human performance: Vol. I. Sensory processes and perception (pp. 21.1-21.57). New York: Wiley.

Thompson, W.B., Willemsen, P., Gooch, A.A., Creem-Regehr, S.H., Loomis, J.M., \&, Beall, A.C. (2004). Does the quality of the computer graphics matter when judging distances in visually immersive environments? Presence, $13,560-571$.

Witt, J.K., Proffitt, D.R., \& Epstein, W. (2004). Perceiving distance: A role of effort and intent. Perception, 33, 577-590

Wu, B., Ooi, T.L., \& He, Z.J. (2004). Perceiving distance accurately by a directional process of integrating ground information. Nature, 428, 73-77.

Received December 15, 2005 Review received January 15, 2006 Accepted February 3, 2006 